\title{
1 Besonderheiten bei Übergewichtigen/Fettleibigen in Chirurgie und Traumatologie
}

\author{
Kaja Ludwig, Sylke Schneider-Koriath und Thomas Westphal
}

Im Zusammenhang mit operativen Eingriffen bei übergewichtigen Patienten müssen sich die beteiligten Ärzte auf zwei grundsätzliche Szenarien einstellen:

Zum einen und überwiegenden Teil werden Operationen im Elektiv- oder Notfallprogramm bei unbehandelten fettleibigen Patienten notwendig, die besondere Aspekte des Managements erfordern. Zum anderen wird in den nächsten Jahren die Zahl der Patienten steigen, die anamnestisch eine durchgeführte Adipositasoperation unterschiedlichster Art aufweisen. An dieser Stelle muss die operative Strategie in Abhängigkeit von Diagnose und Ziel der aktuell durchzuführenden Operation berücksichtigen, dass das Ergebnis der Adipositasoperation zumindest bei abdominalchirurgischen Eingriffen nicht gefährdet wird (z.B. im Zusammenhang mit einer notwendigen Operation wegen einer Sigmadivertikulitis) oder aber unter Umständen in eine normalanatomische Situation rückverwandelt werden muss (z.B. bei einem späterhin diagnostizierten Pankreaskarzinom). Im Hinblick auf notwendige unfallchirurgische oder orthopädische Eingriffe beschränken sich die Maßnahmen zumeist auf perioperative Besonderheiten bei Transport, Lagerung, ggf. OPTechnik und gewichtsadaptierter Medikations- und Mobilisationsunterstützung.

\subsection{Instrumentell-apparative Ausstattung}

Da der Anteil an übergewichtigen Patienten in den letzten Jahren in Deutschland dramatisch zugenommen hat, stehen inzwischen die Krankenhäuser al- ler Versorgungsstufen auch außerhalb adipositaschirurgischer Zentren vor dem Problem, dass sie sich auf diese Patientengruppe einrichten müssen. Bislang enden die TÜV-Zertifikate für Krankenhausbetten und -mobiliar im überwiegenden Fall bei einer Belastungsgrenze von 100-15o kg. Ebenso sind derzeit über $80 \%$ der in Betrieb befindlichen Computertomografen auf eine Belastungsgrenze von $150 \mathrm{~kg}$ limitiert, sodass der phasengerechte Transport des Untersuchungstisches bei Patienten mit einem darüber liegenden Körpergewicht zu schlechten oder fehlerhaften diagnostischen Aussagen führt - wenn sie denn überhaupt in den Röhrenausschnitt passen. Eine MRT-Untersuchung ist aus diesem Grund gegenwärtig vielerorts überhaupt nicht durchführbar.

Für die Krankenhäuser ist es somit zukünftig erforderlich, sich in einem gewissen Umfang auf diese Veränderungen einzustellen. Sicherlich wird es zunächst ausreichend sein, einen Pool an Patientenbetten und -mobiliar einzurichten, das über eine jeweilige Tragfähigkeit von max. 180-200 kg verfügt. Diese Spezialbetten weisen unterstützend elektronische Lagerungshilfen auf und übersteigen im Regelfall in ihrer Ausladung die Weite bisher gültiger StandardTürausschnitte. Ebenso müssen die baulichen Maßnahmen berücksichtigen, dass z.B. die Tragfähigkeit der Patiententoiletten, die Gründung von Schwerlastsäulen im OP oder bei diagnostischen Großgeräten eine besondere Beachtung erfordert. Die weitere Behandlungskette umfasst dann natürlich konsekutiv 


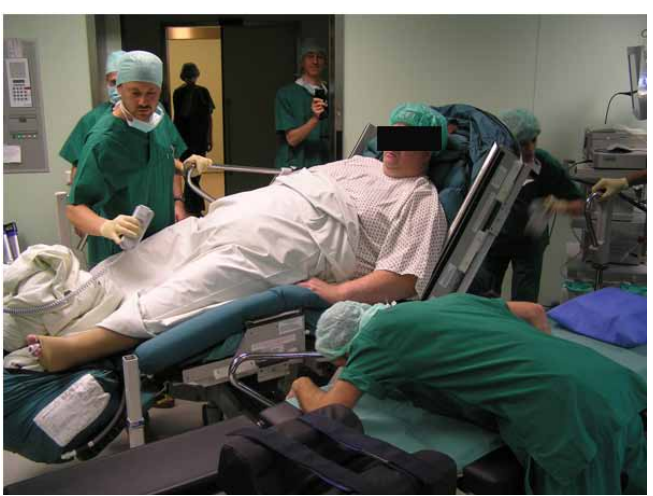

Abb. 1 Patient (228 kg, BMI 71) vor Umlagerung aus einem Spezialbett auf den Operationstisch. Es ist ein höherer Personalaufwand zu beachten.

Mobilisationshilfen (z.B. Rollator), Monitoringausstattungen (z.B. Blutdruckmanschetten) und vieles mehr bis hin zu XXL-Patientenwäsche. Alternativ besteht natürlich immer die Möglichkeit, in Einzelfällen zumindest Spezialbetten o.ä. über Fachfirmen zu leasen, wobei Betten mit einer Tragfähigkeit $>250 \mathrm{~kg}$ nochmals einen deutlich höheren Raumbedarf benötigen.

Im Operationssaal empfiehlt sich die Ausstattung wenigstens eines Saales mit einer Schwerlastsäule bis $350 \mathrm{~kg}$. Es ist dabei zu beachten, dass zumindest im Zusammenhang mit laparoskopischen Eingriffen z.T. extreme Lagerungspositionen notwendig sind, um den äußeren Fettmantel zu überwinden und eine entsprechende Übersicht im Abdominalraum zu gewinnen. Die daraus resultierenden Scherkräfte am Trägersystem des OP-Tisches machen die hohe Traglast notwendig. Ebenso werden mitunter zusätzliche am Tisch arretierbare Lagerungshilfen benötigt, welche gleichermaßen eine ausreichende Stabilität, Umfangsaufnahme und Polsterung aufweisen müssen (s. Abb. 1 und 2).

Spezielle Anforderungen werden natürlich auch an das Operationsinstrumentarium gestellt. Es ist ratsam, zumindest einen Satz überlanger (mind. $45 \mathrm{~mm}$ Schaftlänge) laparoskopischer Instrumente und Trokare vorzuhalten. Sollten - was möglichst zu vermeiden ist - konventionell offene Operationen notwendig werden, ist eine adaptierter Haken-, Sperrer- und Instrumentensatz ebenfalls hilfreich.

Nicht zuletzt muss bei der operativen Behandlung von schwer übergewichtigen Patienten berücksichtigt werden, dass bei möglichen Störungen im postoperativen Verlauf viele sonst übliche diagnos-

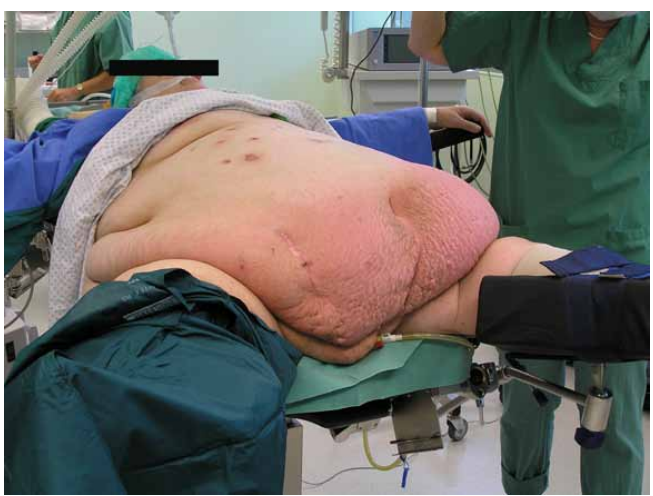

Abb. 2 Patientin (174 kg, BMI 69) nach Lagerung auf dem Operationstisch zur laparoskopischen Dickdarmresektion. Im Hinblick auf mögliche Wundkomplikationen und Lagerungsschäden sind die Veränderungen (Lymphödem, Hauteffloreszenzen) zu beachten und erfordern abweichende Trokarpositionen.

tische Verfahren nur zu einer ungenügend akkuraten Aussage kommen (z.B. KM-Röntgen, Ultraschall). An dieser Stelle rückt die eingangs erwähnte KM-verstärkte CT-Diagnostik in den zentralen Fokus, da ansonsten lediglich die klinische Einschätzung zur Beurteilung eines möglichen Komplikationsausmaßes bleibt, was haftungsrechtlich nicht in jedem Fall ohne Brisanz zu bewerkstelligen ist.

Vor einer notwendigen operativen Behandlung ex-
trem übergewichtiger Patienten müssen alle Aspekte
des perioperativen Managements bedacht werden.
Wenn bestimmte apparativ-instrumentelle Ausrüs-
tungsteile der Klinik nicht vorhanden sind und im Be-
darfsfall auch nicht per Leasing bereitgestellt wer-
den, sollte auch bei vermeintlich banalen Operatio-
nen eine Vorstellung des Patienten in entsprechend
ausgerüsteten Partnerkliniken geprüft werden.

\subsection{Perioperatives Management}

Die morbide Adipositas ist mit bis zu 30 Komorbiditäten vergesellschaftet. Folglich muss einer exakten präoperativen Risikoevaluierung vor jeder Operation Rechnung getragen werden. Im Vordergrund stehen dabei natürlich im Bedarfsfall kardiologische und endokrinologische respektive diabetologische Empfehlungen der jeweiligen Fachkollegen. Es ist darüber hinaus beachtenswert, dass zwischen Übergewicht und psychologischen Störungen eine enge Bezie- 
hung besteht, sodass in Einzelfällen eine perioperativ begleitende psychologische Betreuung der Patienten notwendig wird (Sullivan et al. 1993).

Übergewicht ist in multivariaten Analysen ein unabhängiger und einer der stärksten Risikofaktoren für das Auftreten thromboembolischer Komplikationen (z.B. tiefe Beinvenenthrombosen) im Umfeld operativer Behandlungen (Cotter et al. 2004; Rocha et al. 2006). Aus diesem Grund ist eine gewichtsadaptierte Prophylaxe mit niedermolekularem Heparin absolut indiziert(s. Kap. IX.1). Gleichermaßen sollte die postoperative Immobilisation so kurz wie möglich gehalten werden, womit sich eine weite Indikationsstellung zur laparoskopischen Operationstechnik ergibt.

\section{Allerdings muss im Umkehrschluss auch beachtet werden, dass sich ein Vorteil minimal-invasiver Ope- rationsverfahren im Hinblick auf die Vermeidung von thromboembolischen Komplikationen immer dann verbraucht, wenn unzureichende instrumen- telle Voraussetzungen und ein ungeübter Zugang zu laparoskopischen Operationen bei stark überge- wichtigen Patienten die Operationsdauer deutlich verlängern.}

Die mitunter extreme Lagerungsposition, verbunden mit operationsbedingten höheren Abdominaldrücken (insbesondere im Bereich der V. cava) sind in diesem Zusammenhang als nachteilig anzusehen.

Eine perioperative Antibiotikaprophylaxe sollte bei Operationen übergewichtiger Patienten großzügig erfolgen. Khoury et al. (2011) konnten kürzlich zeigen, dass ein doppelt so hohes Risiko für Wundinfektionen nach laparoskopischer Intestinalchirurgie für übergewichtige gegenüber normalgewichtigen Patienten besteht (10,6\% vs. 4,8\%, p <0,002). Gleichermaßen war die allgemeine Morbidität, speziell im Hinblick auf pulmonale Komplikationen und Harnwegsinfektionen in der Adipositasgruppe tendenziell erhöht (31,5\% vs. $24,2 \%$ ) (Khoury et al. 2011). Primär jedoch erfolgt die Minimierung des Wundinfektionsrisikos über die Wahl der operativen Technik. Im Vergleich von offenen und laparoskopischen Operationen ermittelten beispielsweise Kaafarani et al. (2010) für den offenen Narbenhernienrepair eine 7 -fach höhere Rate an Wundinfektionen $(22,1 \%$ vs. $3,4 \%$, $\mathrm{p}<0,002)$. Für die Appendektomie konnten Li et al. (2004) in einer Metaanalyse über 31 Studien nachweisen, dass bei minimal-invasiver OP-Technik die Wundinfektionsrate halbiert werden konnte $(3,8 \%$ vs. $8,4 \%$, OR o, 45 ).

\subsection{Operatives Management in der Abdominalchirurgie}

Grundsätzlich richtet sich die operative Strategie nach der Art der zu behandelnden Erkrankung und der jeweiligen personellen Expertise im Hinblick auf die Möglichkeiten zur Durchführung laparoskopischer Eingriffe.

Eine Reihe von Studien aus der Adipositaschirurgie konnten zeigen, dass insbesondere bei übergewichtigen Patienten der Einsatz minimal-invasiver Techniken zu deutlichen Vorteilen für die Patienten führt (de Witt et al. 1999; Marema et al. 2005; Nguyen et al. 2000).

Aus diesem Grunde ist der Anteil der laparoskopischen Operationen in diesem Bereich zwischen 1999 und 2004 von $10 \%$ auf über $90 \%$ angestiegen (Benotti et al. 2004). Die Vorteile liegen in einer signifikanten Verminderung postoperativer Komplikationen, geringerem postoperativen Schmerzmittelbedarf, einer schnelleren Mobilisation sowie postoperativen Erholung und letztlich einer deutlich schnelleren Rekonvaleszenz. Gleichermaßen sollten diese nachgewiesenen Vorteile auch bei notwendigen anderweitigen Operationen für übergewichtige Patienten genutzt werden. Es ist in diesem Zusammenhang immer darauf zu achten, dass der Bauchnabel als sonst übliche Orientierungshilfe bei stark übergewichtigen Patienten nicht verwertbar ist und koinzidenziell fast 50\% der Betroffenen eine begleitende Hernie aufweisen. Besser sind als Orientierungshilfen nach entsprechender Lagerung der Rippenbogen bzw. die Spinae iliacae anteriores geeignet. Darüber hinaus sollte beachtet werden, dass die fettleibige Bauchdecke im ventralen Anteil nach Lagerung deutlich dünner ist als in der Flanke. Daraus ergibt sich für die notwendigen Trokarpositionen, dass mittelliniennah im Regelfall Standardlängen ausreichend sind. Bei Trokarpositionen im lateralen Anteil werden $u . U$. Überlängen benötigt.

Ein ganz entscheidender Vorteil der laparoskopischen Technik liegt neben der Senkung des Wundinfektionsrisikos in der Minimierung der Narbenhernienrate. Laurent et al. (2008) konnten zeigen, dass im Vergleich der offenen zur laparoskopischen Rektumresektion die Rate der Narbenhernien in der offenen Gruppe fünf Jahre postoperativ fast dreifach höher war ( $33 \%$ vs. $13 \%$, p < o,oo1). Redundante Daten finden sich für nahezu alle anderen abdomialchirurgischen Eingriffe, z.B. für Sigmaresektionen (14,7\% vs. 
$3,4 \%$ ) oder Ileozökalresektionen bei M. Crohn (14\% vs. 4\%) (Andersen et al. 2008; Stocchi et al. 2008).

\subsection{Operatives Management in der Unfallchirurgie und Orthopädie}

Die eingriffsspezifische Lagerung muss sowohl geplante Zugangswege, deren eventuell notwendige Erweiterung als auch die spezifischen anatomischen Besonderheiten berücksichtigen. So ist bei übergewichtigen Patienten ein höheres Risiko lagerungsbedingter Komplikationen bekannt, sodass gerade auf die Polsterung gefährdeter Regionen besonderes Augenmerk gerichtet werden muss. Unter Umständen sind zusätzliche Polstermaterialien, aber auch Abdecktücher vorzuhalten. Da durch die Körpermasse Beeinträchtigungen der intraoperativen Bildgebung zu erwarten sind, muss die Bildgebung mit einem Röntgenbildverstärker unbedingt zu Beginn der Operation getestet werden (Harrington 1997; Strauss et al. 2007).

Die Zugangswege weichen nicht von denen bei normalgewichtigen Patienten ab. Zu berücksichtigen ist jedoch, dass das Erreichen des eigentlichen Operationssitus und die Darstellung der Anatomie erschwert sein können, sodass Erweiterungen der Zugangswege, ggf. alternative Zugänge, einzuplanen sind.

Insbesondere sind adäquate Instrumente, wie große Wundhaken, lange Scheren, Pinzetten usw. vorzuhalten. Angesichts des ohnehin erhöhten Risikos für die Entstehung von Wundkomplikationen bei fettleibigen Patienten sollten keine Kompromisse bei der Wahl des Operationszuganges und der Auswahl der Instrumente eingegangen werden.

Wie auch in der Viszeralchirurgie ist endoskopischen (arthroskopischen) Verfahren, wo immer möglich, der Vorzug zu geben. Auch hier sind längere Instrumente nützlich.

Die osteosynthetische Versorgung muss bei adipösen Patienten den bekanntermaßen schwereren Frakturmustern, der schlechten Mobilisierbarkeit und der Komplikationshäufigkeit Rechnung tragen (Kushawa u. Garland 1998). So sollten immer die stabilsten Implantate ausgewählt werden, eventuell muss die Anzahl der üblicherweise zur Verankerung von Platten nötigen Schraubenanzahl erhöht werden. Winkelstabile Implantate bieten im osteoporotischen Knochen Stabilitätsvorteile (Jones 2011; Lazar et al. 2010). Im Einzelfall kann eine Augmentation mit Zementen erwogen werden. Bei intramedullären Implantaten ist die Ausnutzung aller Verriegelungsoptionen zu empfehlen. An lasttragenden Extremitätenabschnitten ist eine möglichst optimale Abstützung der Fragmente anzustreben.

\subsection{Sonderfall - Patienten mit vorausgegangenen Adipositasoperationen}

Mit der weiteren Verbreitung der Adipositaschirurgie in Deutschland ist damit zu rechnen, dass mehr oder minder übergewichtige Patienten nach vorausgegangener Adipositasoperation erneut behandelt oder operiert werden müssen. Vollkommen unproblematisch sind in diesem Zusammenhang notwendige Eingriffe an der Gallenblase oder wegen einer akuten Appendizitis. Nachdem über viele Jahre hinweg die Cholezystektomie und Appendektomie im Zusammenhang mit einer Adipositasoperation umfänglich mit ausgeführt wurde, erfolgen heute diese Eingriffserweiterungen eigentlich nur noch bei bilio-pankreatischen Diversionsoperationen (BPD m/o duodenalem Switch) oder bei entsprechender Symptomatik. Es ist also ratsam, vor geplanter Operation - wenn möglich - Informationen zur Vor-Operation zu sammeln. Ebenso erscheinen notwendige Eingriffe am Dick- und Enddarm weniger problembelastet und können mit Ausnahme der Diversionsoperationen nach Standardvorgehen erfolgen.

\section{Im Gegensatz dazu müssen Operationen bei gutarti- gen Erkrankungen im Bereich des Magens respekti- ve Dünndarms in Abhängigkeit von der Vor-Operation subtil geplant werden, um das System der Restriktion oder Malabsorption nicht zu gefährden.}

Bei Diagnose von bösartigen Erkrankungen (obgleich das Risiko nach Adipositasoperation deutlich verringert ist) muss ggf. eine Rückumwandlung in die natürliche Passage erfolgen. In diesen Situationen sollte grundsätzlich eine intensive Diskussion mit einem erfahrenen Adipositaschirurgen, u.U. auch eine Beteiligung desselbigen an der Operation erfolgen.

Entscheidend für diagnostische und therapeutische Handlungswege nach vorausgegangener Adipositasoperation ist das typische Komplikationsspektrum in Abhängigkeit von der zurückliegenden Zeitdauer. So konnten Zingmond et al. (2005) bei einer Analyse nach über 6o.ooo Magen-Bypassoperationen zeigen, dass über 90\% der zur stationären Aufnahme führen- 
den Komplikationen innerhalb von 3 Monaten auftraten und in 90\% der vorangegangenen Bypassoperation zuzuordnen waren. Lediglich in 10\% war eine Intervention erforderlich. $35 \%$ der Patienten wurden in nicht-spezialisierten wohnortnahen Krankenhäusern behandelt (Zingmond et al. 2005). Wenn möglich, sollte also auch in diesen Fällen eine Behandlung in enger Abstimmung mit den erstoperierenden Kliniken erfolgen.

\section{Zusammenfassung}

Notfallmäßige oder elektive Operationen bei Patienten mit (schwerer) Adipositas erfordern ein spezielles Management und sind mit besonderen Komplikationsmöglichkeiten belastet. Selbst "banale“ Operationen sollten daher wohl geplant und - wenn möglich - in speziellen Zentren durchgeführt werden. Bei viszeralchirurgischen Operationen von Patienten, die sich zuvor keiner bariatrischen OP unterzogen haben, ist dringend die vorherige Kontaktaufnahme mit dem entsprechenden Team ratsam.

\section{Literatur}

Andersen LP, Klein M, Geogenur I, Rosenberg I (2008) Incisional hernia after open versus laparoscopic sigmoid resection. Surg Endosc 22, 2026-2029

Cotter SA, Cantrell W, Fisher B, Shopnick R (2005) Efficacy of venous thromboembolism prophylaxis in morbidly obese patients undergoing gastric bypass surgery. Obes Surg 15, 1316-1320

Harrington KD (1997) Orthopedic surgical management of skeletal complications of malignancy. Cancer 80, 1614-1627

Iones CB (2011) Management of upper extremity injuries in obese patients. Orthop Clin North Am 42, 11-19

Khoury W, Stocchi L, Geisler D (2011) Outcomes after laparoscopic intestinal resection in obese versus non-obese patients. B/S 98 , 293-298
Kushawa VP, Garland DG (1998) Extremity fractures in patients with a traumatic brain injury. I Am Acad Orthop Surg 6, 298-307

Laurent C, Leblanc F, Bretagnol F, Capdepont M, Rullier E (2008) Long-term wound advantages oft he laparoscopic approach in rectal cancer. Br | Surg 95, 903-908

Lazar MA, Plocher EK, Egol KA (2010) Obesity and ist relationship with pelvic and lower-extremity othopedic trauma. Am J Orthop 39, 175-182

Li X, Zhang I, Sang L, Zhang W, Chu Z, Li X Liu Y (2010) Laparoscopic versus conventional appendectomy - a meta-analysis of randomized controlled trials. BMC Gastroenterol 10, 129-136

Marema RT, Perez M, Buffington CK (2005) Comparison of benefits and complications between laparoscopic and open Roux-en-Y gastric bypass surgeries. Surg Endosc 19, 525-530

Nguyen NT, Ho HS, Palmer LS, Wolfe BM (2000) A comparison study of laparoscopic versus open gastric bypass for morbid obesity. | Am Coll Surg 191, 149-157

Rocha AT, de Vasconcellos AG, da Luz Neto ER, Araujo ES, Lopez AA (2006) Risk of venous thromboembolism and efficacy of thromboprophylaxis in hospitalized obese medical patients and in obese patients undergoing bariatric surgery. Obes Surg 16, 1645-1655

Stocchi L, Milsom JW, Fazio VW (2008) Long-term outcomes of laparoscopic versus open ileocolic resection for Crohn's disease: follow-up of a prospective randomized trial. Surgery 144, 622627

Strauss EJ, Frank JB, Walsh M, Koval KJ, Egol KA (2007) Does obesity influence the outcome after operative treatment of ankle fractures? | Bone loint Surg Br 89, 794-798

Sullivan M, Karlsson I, Sjöström L, Backman I, Bengtsson C, Bouchard C, Dahlgren S, Jonsson E, Larsson B, Lindstedt S, Näslund I, Olbe L, Wedel H (1993) Swedish obese subjects (SOS) an intervention study of obesity. Baseline evaluation of health an psychological functioning in the first 1743 subjects examined. Int I Obes Relat Metab Disord 17, 503-512

de Wit LT, Mathus-Vliegen L, Hey C, Rademaker B, Gouma DJ, Obertop $H$ (1999) Open versus laparoscopic adjustable silicone gastric banding: a prospective randomized trial for treatment of morbid obesity. Ann Surg 230, 800-807

Zingmond DS, McGory ML, Ko CY (2005) Hospitalization before and after gastric bypass surgery. JAMA 294, 1918-1924

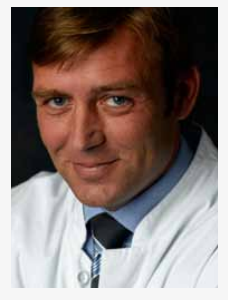

Prof. Dr. med. Kaja Ludwig

Nach Abschluss des Medizinstudiums 1989 zunächst Facharztweiterbildung in Seehausen sowie an der Chirurgischen Universitätsklinik in Greifswald. Die Promotion erfolgte 1996. 2002 Habilitation und 2008 apl-Professur an der Universität Greifswald. Seit 1997 zunächst Oberarzt an der Chirurgischen Universitätsklinik in Greifswald, ab 2000 gf. Oberarzt am Klinikum Südstadt Rostock, seit 2003 Chefarzt der Klinik für Chirurgie am Klinikum Südstadt Rostock. 


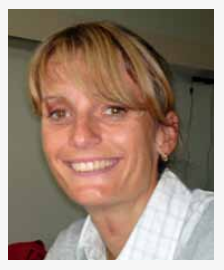

\section{Dr. med. Sylke Schneider-Koriath}

Nach dem Medizinstudium an der Universität Rostock von 1992-1998 mit Aufenthalten von 04/98-09/98 in Kapstadt und Durban arbeitete sie ab 1999 am Klinikum Freising in den Abteilungen für Allgemein-, Thorax- und Gefäßchirurgie sowie Unfall-und Wiederherstellungschirurgie. 05.05.2004 Ablegen der Facharztprüfung Chirurgie.

Seit 01.07.2005 am Klinikum Südstadt Rostock, Klinik für Chirurgie, Abteilung für Allgemein-, Viszeral- und Gefäßchirurgie tätig. 01.10.2008 Schwerpunktbezeichnung Viszeralchirurgie. Seit 01.02.2009 Oberärztin der Klinik für Chirurgie.

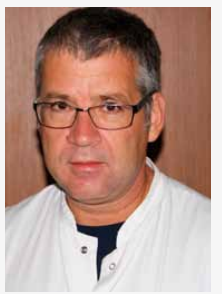

\section{Dr. med. Thomas Westphal}

Nach dem Studium an der Universität Rostock begann er 1990 seine Ausbildung zum Facharzt für Chirurgie im damaligen Kreiskrankenhaus Weißwasser. 1996 wechselte er in die Klinik für Unfallchirurgie des Universitätsklinikums der Otto-von-Guericke-Universität Magdeburg. 1997 Anerkennung als Facharzt für Chirurgie, 1998 Promotion, 2000 Anerkennung im Schwerpunkt Unfallchirurgie und Oberarzt. 2005 Wechsel in die Klinik für Chirurgie des Klinkums Südstadt Rostock als leitender Arzt der Abteilung Unfallchirurgie/Orthopädie, seit 2010 Chefarzt. 2007 Facharzt für Orthopädie und Unfallchirurgie, 2008 Zusatzbezeichnung Spezielle Unfallchirurgie. 